\title{
Group Structural Realism
}

\author{
Bryan W. Roberts*
}

June 26, 2009

\begin{abstract}
We present a precise form of structural realism, called group structural realism, which identifies 'structure' in quantum theory with symmetry groups. However, working out the details of this view actually illuminates a major problem for structural realism; namely, a structure can itself have structure. This paper argues that, once a precise characterization of structure is given, the 'metaphysical hierarchy' on which group structural realism rests is overly extravagant and ultimately unmotivated.
\end{abstract}

1 Introduction

2 What Is (Group) Structural Realism?

2.1 The Basic Picture

2.2 Wigner's Legacy

2.3 Accounting for Theory Change

3 The 'Higher Structures' Problem

3.1 The Structure of a Structure

3.2 Higher Structures and Theory Change

3.3 Any Way Out for the Groupies

3.3.1 Chopping Down the Tower

3.3.2 Playing Favorites

3.3.3 Adopting the Whole Shebang

4 Conclusion

5 Appendix

*Email: bwr6@pitt.edu. Web: www.pitt.edu/〜bwr6 


\section{Introduction}

There is a part of structural realism that is basically correct, and a basic consequence of structural realism that is deeply implausible. The goal of this paper is to clear the muck around what structural realism gets right, in order to bring out a new difficulty facing the view.

The thesis of the paper has both a positive and a negative part. The positive part is this:

- Structural realism has a solid basis in quantum physics, which provides valuable insight into both the nature of measurable quantities, as well as into what's preserved when theories change.

In particular, structural realists have been correct to insist on the fundamental importance of symmetry groups in the foundations of quantum theory. We will draw out the precise view that lurks behind this insistence, and give it a name: group structural realism.

Group structural realism will provide us with a concrete viewpoint from which to survey a general difficulty for structural realism. The statement of this problem is the negative part of the thesis:

- The 'metaphysical hierarchy' on which structural realism rests is overly extravagant at best, and arbitrary worst.

The paper is organized into two parts: first, an exposition of group structural realism, in which we develop the positive thesis; and second, a critique of structural realism, in which we develop the negative. The first part begins with a general picture of group structural realism in contrast with related accounts. We demonstrate the connection this view provides between structure and measurable quantities, and then offer some positive remarks about theory change. The second part illustrates how structural realism is troubled by the existence of 'higher' structures. We show that in the case of group structural realism, these higher structures also provide a positive account of theory change, and finally argue that there appears to be no well-motivated way to incorporate them into the structural realist picture. 


\section{What Is (Group) Structural Realism?}

\subsection{The Basic Picture}

John Worrall (1989) suggested that some notion of 'structure' might allow scientific realists to overcome the problems posed by theory change and the pessimistic meta-induction. This view was later developed by James Ladyman (1998) into an explicit metaphysical thesis, which begins by 'taking structure to be primitive and ontologically subsistent' (Ladyman 1998, pp. 420). Now, many realists are advocating an ontology that gives structural relations a more central role than individual objects ${ }^{1}$.

The positive claim of structural realism differs greatly from author to author. Notably, structural realists break down into 'eliminativist' and 'non-eliminativist' accounts. While the former eliminates objects (claiming that only structure exists), the latter merely demotes (but does not eliminate) objects to a lesser metaphysical status. However, there is one core assumption that most accounts of structural realism (as a metaphysical view) do share:

The Structural Realist Hierarchy. The existing entities described by a scientific theory are organized into a hierarchy, in which 'structure' occupies the top, most fundamental position.

The eliminativist takes this hierarchy to contain only structure (or perhaps even just one structure, making the hierarchy trivial). The non-eliminativist allows the hierarchy to contain objects in some low-status position. In the latter case, what it means to be 'fundamental' in a hierarchy of entities can be cashed out in various ways. For example, Ladyman and Ross characterize it using the notion of supervenience on properties:

Ontic Structural Realism (OSR) is the view that the world has an objective modal structure that is ontologically fundamental, in the sense of not supervening on the intrinsic properties of a set of individuals. According to OSR, even the identity and individuality of objects depends on the relational structure of the world. (Ladyman and Ross 2007, pp. 130.)

Unfortunately, the precise notion of priority that's at stake here remains obscure. One can always resort to analogies: the droplets of paint on a canvas might be considered more fundamental than

\footnotetext{
${ }^{1}$ For an overview, see (Ladyman 2009).
} 
the images in the painting, because the images supervene on the droplets. Similarly; the atoms in a molecule are more fundamental than the molecule itself, which is more fundamental than the substance. Structural realists argue that at the very top, at the most fundamental layer of this hierarchy, there is only structure. But these analogies break down: images in a painting certainly cannot exist in the absence of paint droplets, but the shadowy existence of structure is much less clear $^{2}$.

Our strategy here will be to grant the structural realist as much as possible on such matters, by leaving open exactly how one might understand 'priority' in a metaphysical hierarchy. Instead, we will assume that a such a notion can be established, and is capable of either

- providing a well-motivated description of the structure that is most fundamental; or

- describing a multiplicity of fundamental structures, which better informs our understanding of what exists,

given the history of our best scientific theories. In the next subsection, we will describe a precise example from quantum theory, which appears to provide a promising way to establish the structural realist hierarchy. Unfortunately, we must later struggle with a dilemma, which suggests that the existence of higher structures thwarts the success of either option.

Why propose a structural realist hierarchy? Broadly speaking, there are two main goals. First: structural realism aims to provide a general, programmatic account of science and scientific discovery. Structural realist accounts of the metaphysics of theory change, such as Ladyman (1998), are canonical examples. Second: structural realism aims to solve specific problems in the interpretation of a theory. For example, it has been proposed as a solution to the problem of identical particles (Ladyman and Ross 2007, §3.1), and to the problem of interpreting spacetime points (Ladyman 1998; Dorato 2000). Which structures are of interest may differ depending on one's goals. To avoid confusion, we will thus focus our attention in this paper on the first goal: for structural realism to provide an improved account of how theories change.

Standing between structural realism and what it endeavors to achieve is the meaning of the word structure. Ladyman and French themselves note that 'Because of the width of its embrace and

\footnotetext{
${ }^{2}$ Kantorovich (2006) has recently argued that some structures can exist even in the absence of particles.
} 
its complex history, defining what is meant by "structure" and characterizing the tendency in general, is problematic' (French and Ladyman 2008, §1). Ladyman and Ross similarly accept the criticism that structural realism may not be well 'worked out.' However, they retort that 'it is far from clear that OSR's rivals are 'worked out' in any sense that OSR isn't' (Ladyman and Ross 2007, pp. $155)$.

Clearly, someone ought to work something out. As a start, I will try to show that, using the resources of group theory and quantum mechanics, a precise characterization of 'structure' can be worked out in as much detail as you like. The specific view that I propose we work out is the following:

Group Structural Realism (GSR). The existing entities described by quantum theory are organized into a hierarchy, in which a particular symmetry group occupies the top, most fundamental position.

Like the structural realist hierarchy set out above, this statement of GSR should be taken as a minimal assumption of the view. Focusing on this assumption allows us to leave the exact nature of a group structure's 'existence' to the individual metaphysician.

GSR has a good deal of precedent among structural realists. For example, Aharon Kantorovich argues for a conception of particle physics in which 'internal symmetry is the deepest layer in the ontological hierarchy,' and in particular, that 'flavor $S U(3)$ symmetry was ontologically prior to hadrons [...] whereas $S U(5)$ is ontologically prior only to baryons' (Kantorovich 2003. pp. 673). Holger Lyre has suggested an account of objects that 'takes the group structure as primarily given, group representations are then construed from this structure and have a mere derivative status' (Lyre 2004, pp. 663). Similarly, Ladyman and Ross argue that, 'elementary particles are hypostatizations of sets of quantities that are invariant under the symmetry groups of particle physics' (Ladyman and Ross 2007, pp. 147).

Unlike many more abstract accounts of structural realism, quantum theory provides GSR with a precise connection to the physical quantities that we actually observe and measure in the lab. In the next section, we will review the physical results that provide this connection. 


\subsection{Wigner's Legacy}

Yuval Ne'eman and Shlomo Sternberg have recorded an old particle physicist's adage:

Ever since the fundamental paper of Wigner on the irreducible representations of the Poincaré group, it has been a (perhaps implicit) definition in physics that an elementary particle 'is' an irreducible representation of the group, $G$, of 'symmetries of nature'. (Ne'eman and Sternberg 1991, pp. 327.)

Despite their abstractness, irreducible unitary representations do seem to satisfy our intuitions about elementary particles. Jonathan Bain suggests two such intuitions: (1) an elementary particle should be uniquely labeled by a mass and a spin parameter (that is, by the eigenvalues of a total 4-momentum and a total 4-angular momentum operator); and (2) a particle should be invariant up to the group of spacetime symmetries, in order to satisfy 'our intuitions concerning the continuity of particle identity through time' (Bain 2000, pp. 402fn). One also wants that, (3) an elementary particle cannot be 'decomposed' into further particles; and (4) a particle should be associated with a set of observables that describe its possible states.

One can now observe: Wigner showed that the irreducible unitary representations of the Poincaré group do indeed satisfy (1) and (3) because of irreducibility; they satisfy (2) because they represent the Poincare group; and, they satisfy (4) because they are unitary ${ }^{3}$.

Although this metaphysical picture of 'particles as representations' is often attributed to Wigner, he does not seem to have advocated it in print. The famous (1939) paper that Ne'emann and Sternberg refer to sets out only to correlate the values of physical magnitudes (the so-called 'quantum numbers') with parameters labeling group representations - in particular, the representations of the spacetime symmetry group. By classifying all the irreducible unitary representations of this group, Wigner is able to identify all the possible labels of mass, spin and parity. This provides a tight connection between a symmetry group of nature, and the measurable properties of a quantum system.

A simple textbook example will help to illustrate this connection. Take a familiar physical property like angular momentum. In a given situation, quantum theory assigns a fixed value to

\footnotetext{
${ }^{3}$ Here's a sketch of how one can obtain (4). The Poincaré group is the product of 1-parameter subgroups. Consider a strongly continuous unitary representation of any such subgroup. Stone's theorem guarantees that this representation is generated by a unique self-adjoint operator $H$. This operator is an observable, in any state space on which $U$ acts.
} 
some aspects of angular momentum, like (say) the total angular momentum of an isolated system. Other aspects, such as 'angular momentum in the $z$-direction,' might (prior to measurement) be assigned a spectrum of values. Wigner's approach allows us to think of physical magnitudes such as these as properties of a symmetry group.

To simplify our example, we can ignore the existence of $\operatorname{spin}^{4}$. One begins with the group $S O(3)$ of continuous rotations about a point. The faithful irreducible representations of $S O(3)$ turn out to be representable by groups of complex-valued matrices of odd dimension $(2 j+1)$, where $j$ is a positive integer. If desired, a given representation can be thought of as acting on, say, the state space of an electron shell around a Hydrogen atom. However, the imagery of this individual object isn't required for our construction. Instead, we can skip directly to defining the total angular momentum $j=(n-1) / 2$, in terms of the dimension $n$ of the representation. The angular momentum operators can then be picked out as elements of the representation, and angular momentum in the $z$-direction can then be defined and shown to have the usual integer-stepped spectrum, $\{-j, \ldots, 0, \ldots, j\}$.

In summary: angular momentum is recovered, with all its expected properties, from facts about a symmetry group; no assumptions about the state $\psi$ of an individual object are needed ${ }^{5}$. The construction achieves roughly what Eddington suggested, that '[i]n fundamental investigations the conception of group-structure appears quite explicitly as the starting point; and nowhere in the subsequent development do we admit material not derived from group structure' (Eddington 1958. pp. 147). That such a development is possible is a fact about the physics. But it is also what paves the way for a reasonable structuralist metaphysics. Wigner's approach is just what is needed to allow the group structural realist to speak safely of properties like angular momentum, without recourse to an ontology of individual objects.

In particular, GSR places group structure at the top of the metaphysical hierarchy. In the example of angular momentum, the fundamental structure is the rotational symmetry group $S O(3)$. A measurable physical magnitude $(j)$ provides information about the group (in this case, it picks out the dimension of the representation). And, if desired, one can proceed further to construct a model of an individual object, like a hydrogen atom - but this kind of construction would be

\footnotetext{
${ }^{4}$ Spin is incorporated just as easily by replacing $S O(3)$ with its double-covering group $S U(2)$ and following the same procedure.

${ }^{5}$ This standard textbook computation was first published in a book by Wigner (1931).
} 
metaphysically 'secondary.'

Recent structural realists have tried to express this kind of situation in terms of invariants. To continue the example: one can think of the rotation group as shifting around the states of an electron shell, through the action of the group on state space ${ }^{6}$. When the states undergo this action, the quantity $j$ (which we associate with a real physical magnitude) remains an invariant quantity.

However, structural realists have encountered some difficulty in describing this action, which is inevitably on something like an electron shell. An object like an electron shell might appear to be 'non-structural.' So, many structural realists have tried to rephrase the situation, with the language of action awkwardly excised. Here's Ladyman: 'Objects are picked out by individuating invariants with respect to the transformations relevant to the context. Thus, on this view, elementary particles are just stets of quantities that are invariant under the symmetry groups of particle physics' (Ladyman 1998, pp. 421). Steven French follows: 'With these invariants understood and represented group theoretically, we arrive at a kind of structural realism which takes structure seriously [...]. Thus the elements themselves, regarded as individuals, have only a heuristic role in allowing for the introduction of structures which then carry the ontological weight' (French 1999. pp. 204).

French and Ladyman's use of the word 'invariant' might be misleading, if one thinks that 'invariance under a group action' is supposed to make sense without anything there to be acted upon! Fortunately, Wigner's legacy provides a more natural approach. One can begin the construction of quantum theory from a symmetry group, and still speak perfectly well about measurable quantities. A measurable quantity like angular momentum $(j)$ is of course derived from a representation space, and one can speak freely about its invariance under the action on that space. The advocate of GSR simply holds that, metaphysically, the most significant feature of this space is that it provides a copy of the rotation group ${ }^{7}-$ and not that it refers to the possible states of an individual object.

Construed this way, GSR leads to some surprisingly informative consequences. Let's think about what it would mean if spacetime had a symmetry group other than the Poincaré group. This new group would have different representations, and would thus allow for different properties

\footnotetext{
${ }^{6}$ In this case, the canonical action of $S O(3)$ on the Hilbert space representation would be $\alpha: S O(3) \times \mathcal{H} \rightarrow \mathcal{H}$ such that $\alpha(R, \psi(\mathbf{x}))=\psi(R \mathbf{x})$, where $\psi(R \mathbf{x})$ denotes the state $\psi(\mathbf{x})$ in the rotated frame.

${ }^{7}$ That is, the group of $(2 j+1) \times(2 j+1)$ matrices constructed for the representation is isomorphic to $S O(3)$.
} 
of quantum systems. On Ne'eman and Sternberg's definition, this means that there are different 'particles.' In fact, that is exactly what Bargmann (1954) and Lévy-leblond (1967) have shown: the Galilei group gives rise to a theory of 'Galilei particles,' which are different (in particular, with respect to the 'mass' parameter) than the usual 'Poincaré particles.'

Let's take another case: what would it mean for nature to admit more symmetries than just those of spacetime? According to GSR, this larger group would provide richer representations, and so in a sense 'more' properties for quantum particles. This is just what is suggested by the study of internal symmetries. For example, Gell-Mann (1961) and Ne'eman (1961) advocated $S U(3)$ as a symmetry group. This led them to a new taxonomy of hadrons (as they are now called), classified according to the irreducible representations of the new symmetry group ${ }^{8}$.

Of course, in building up a useful quantum theory, many mathematical objects besides groups come into play: vector spaces, commutation relations, Hermitian forms, and on and on. GSR need not deny this. Rather, GSR implies that out of all these tools, group structure is the one of central metaphysical importance. Other realists might propose a different foundation for the theory, perhaps by arguing (with Geoffrey Sewell) that, 'theories of such systems should be based on the algebraic structure of their observables, rather than on particular representations thereof' (Sewell 2002, pp. 18) ${ }^{9}$. So, why choose GSR over all these other options? Here, the two overarching aims of structural realism come into play: groups are thought to do a better job of providing a general programmatic account of science, or of solving specific problems in the interpretation of scientific theory.

While a general overview of all of these aims is outside the scope of this exposition ${ }^{10}$, we can make some progress with a discussion of how GSR satisfies one popular goal for structural realism as a metaphysical view ${ }^{11}$ : to describe a realist metaphysics that gets preserved as theories change. Let us now turn to this goal.

\footnotetext{
${ }^{8}$ For a structural realist account of internal symmetries, see (Kantorovich 2003).

${ }^{9}$ Of course, an algebra is also a group, so Sewell's suggestion is perhaps best viewed as a closely related cousin to GSR.

${ }^{10}$ See (Ladyman and Ross 2007) for a broad start.

${ }^{11}$ In this paper, we will not be concerned with advocates of epistemic forms of structural realism, who may not endorse to any particular metaphysical view about structure.
} 


\subsection{Accounting for Theory Change}

Can groups do a better job at surviving theory change than individual objects? Weyl certainly seemed to think so, remarking that, 'We may well expect that it is just this part of quantum physics that is most deserving of a lasting place' (Weyl 1950 pp. xxi). More recently, Holger Lyre has argued that 'there is a considerable element of retention of group structure and its embedding into a larger framework which makes the scientific progress much less discontinuous as it looks on the level of objects' (Lyre 2004, pp. 664). One observation to motivate these claims is this: groups are often insensitive to a change in underlying set. So, it's possible for the group structure of an early scientific theory to be preserved in a later theory, even if the descriptions of objects are not.

Here's a toy example: Imagine that some theory leads us to propose the existence of a cube. Suppose that later, we discover that there is no cube, but rather an octahedron. This theory was wrong about what kinds of objects exist. However, it was right about the group-structure, since cubes and octahedrons have the same symmetry group (rotations of $\pi / 2$ about appropriate axes preserve the orientations of both objects; so do flips about an appropriate plane). So, if we were betting on which item would be preserved under theory change, a bet on groups would have won out over a bet on objects.
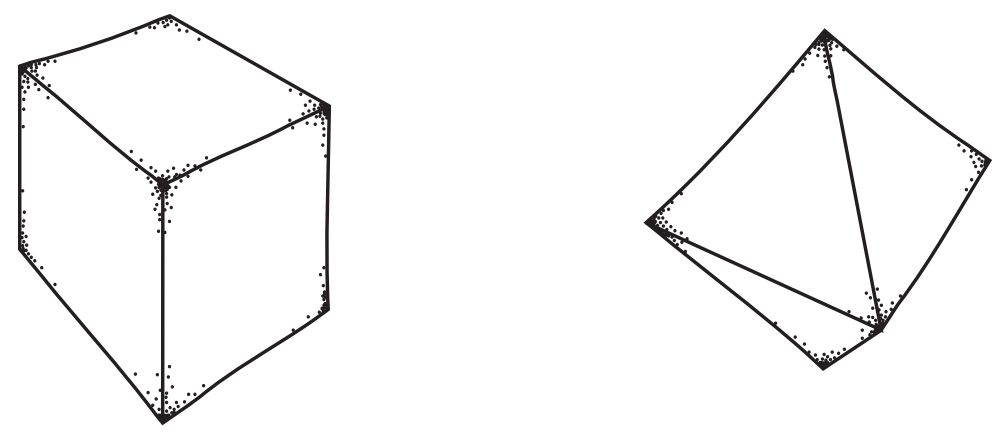

Figure 1: An cube and an octahedron are two different objects with the same symmetry group.

Recently, French and Ladyman have suggested a more realistic examples of this: 'it is part of the structure of Newtonian mechanics that the laws of physics are invariant under the Galilean transformations, and the latter are recovered in approximate form as part of the structure of relativistic physics' (French and Ladyman 2008, §4).

It's worth expanding on this example. As we noted above, Wigner's legacy allows for a the- 
ory of 'Galilei particles.' However, the group of Galilei transformations predicts the wrong kinds of particles (in particular, the wrong momentum eigenvalues), as well as the wrong commutation relations ${ }^{12}$. Consequently, in the transition to the Poincare group, the taxonomy of fundamental particles changed. However, 'Galilean particles' do happen to have the right angular momentum quantum numbers - they allow for the possibility of spin, for example. A realist about particles has little to say about this fact. But GSR can actually provide an explanation: it is because rotation group is what's metaphysically fundamental about angular momentum, and the rotation group was preserved in the transition from the Galilei to the Poincare group, as a subgroup of each. As for the Galilei group as a whole, one might say that it was also preserved in approximate form, in low-velocity regimes.

In fact, $S O(3)$ provides yet another precise example of preservation under theory change. With the discovery of spin, the traditional realist should seemingly admit that a new kind of particle was discovered, signifying a discontinuity over theory change. But, according to GSR, the important change was really the extension of the symmetry group $S O(3)$ to a larger group, $S U(2)$. The latter is the correct rotation group for a quantum theory of spin, because it admits $j=1 / 2$-integer representations. However, $S O(3)$ is not rejected in this correction - it is preserved as a subgroup of $S U(2)^{13}$.

The point of these examples, for the budding structural realist, is to suggest that group structures - not individual objects, and not even algebras of observables - are the superior candidates for the survival of theory change. If this turns out to be right, then GSR not only provides a natural, precise example of structural realism; it also stands a promising chance of satisfying the original, 'pessimistic meta-induction' motivation for structural realism.

\footnotetext{
${ }^{12}$ See especially (Bargmann 1954 §6).

${ }^{13} S U(2)$ is the double-covering group of $S O(3)$, and is thus isomorphic to the semi-direct product $S O(3) \ltimes\{1,-1\}$. It follows that $S O(3)$ is a subgroup of $S U(2)$.
} 


\section{The 'Higher Structures' Problem}

\subsection{The Structure of a Structure}

French and Ladyman have noted the possibility that a structure might itself be describable in structural terms; they note that this 'hyperstructuralist route' faces the worrisome possibility of an infinite regress. The discussion is embedded in a response to Psillos (2006, 569-570), who argues that structuralists will have some difficulties in accounting for causal properties. Here is part of their response:

perhaps the most intuitively plausible form of structuralism is precisely one according to which objects and their properties are metaphysically dissolved into a multilayered network of relations, where certain of these relations are causally empowered and where this empowerment, for want of a better word, is inherent to the relation. is that inherent empowerment non-structural? Yes, in the sense that it is not itself a structure or describable in structural terms (if it were so describable an obvious regress would threaten); no, in the sense that it is another aspect of the world structure. And again, even if one were to go the hypestructuralist route, it is not clear why moving up a level, as it were, would render causal powers as nothing but formal structure. (French and Ladyman 2008, §6.)

The problem that French and Ladyman are addressing applies to much more than causal structure alone (setting aside what that might be). The worry rather derives from the fact that a relation is an amazingly general notion. Relations can describe not only objects, but also other relations. Consequently, it is a very general fact about a structure (which is made up of relations) that it itself often admits a structure (made up of relations between the relations). This level of generality is crucial to almost everything that structural realism sets out to achieve. A 'structure' is so general that it can describe two very different objects. (Hence, structure is more apt to survive theory change, and so on.) A structure is so general that it can even describe other structures - it can even describe its own structure!

If $S$ is a structure, what is the status of the structure of $S$ itself? Presumably, if the original structure $S$ were at the top of the metaphysical hierarchy, then the structure of $S$ must have a 
secondary, derivative status - much like the status of individual objects. But then, it's not obvious why we should choose to place one structure at the top of the hierarchy over the other. This raises a problem for structural realism, in the form of a dilemma. On the one horn, we would like to choose just one structure to be at the top of our metaphysical hierarchy. But it is unlikely that we will be able to give a well-motivated reason to choose between a structure $S$, and the structure of $S$ itself. This pushes us to the other horn: we must promote the whole shebang, both $S$ and the structure of $S$, to a metaphysically 'fundamental' status. But this account of metaphysics, if one can even make sense of what counts as the 'whole shebang,' leads to an much more complex hierarchy, which need not satisfy the aims of structural realism.

Of course, one can declare outright (as French and Ladyman do) that a given structure of interest 'is not itself a structure describable in structural terms.' However, the status of this claim depends on which structure one is talking about. In the case of GSR, the claim is simply false: there is an important sense in which symmetry groups are describable terms of their own symmetry group structure.

The 'symmetry group structure' describing a group $G$ itself is called the automorphism group, Aut $G$. An automorphism $\alpha$ of a group $G$ is a mapping from $G$ to itself that preserves group structure $^{14}$. The group Aut $G$ is formed by collecting together the set of all such automorphisms, and taking the binary operation to be functional composition. Now, to see in what sense Aut $G$ describes the 'symmetries' of $G$, consider the following analogy with the Wigner-approach to GSR.

Begin by presenting $S O(3)$ as the group of rotations $R_{\mathbf{x}}(\theta), R_{\mathbf{y}}(\theta), R_{\mathbf{z}}(\theta)$ of a sphere, where $\mathbf{x}, \mathbf{y}$ and $\mathbf{z}$ are orthogonal axes of rotation. Then there is an automorphism of $S O(3)$ formed by a smooth rotation of these axes, by mapping each rotation $R_{\mathbf{x}}(\theta)$ to a rotation $\alpha\left(R_{\mathbf{x}}(\theta)\right)$ about a new axis ${ }^{15}$. The class of all such automorphisms forms a subgroup of Aut $S O(3)$, which is visibly isomorphic to $S O(3)$ itself. The rest of the automorphisms involve an orthogonal transformation of the axes that is not accessible by a smooth rotation, and so the full automorphism group turns out to be given by the semi-direct product $S O(3) \rtimes\{-1,1\} \cong$ Aut $S O(3)$. The situation is illustrated

\footnotetext{
${ }^{14}$ More precisely, an automorphism on a group $(X, \circ)$ is a bijection $\alpha: X \rightarrow X$ such that $\alpha\left(x_{1} \circ x_{2}\right)=\alpha\left(x_{1}\right) \circ$ $\alpha\left(x_{2}\right)$.

${ }^{15}$ In particular, this is an inner automorphism. For any fixed $\bar{R} \in S O(3)$, an inner automorphism can be defined by the mapping $\alpha_{\bar{R}}: S O(3) \rightarrow S O(3)$ such that $\alpha_{\bar{R}}(R)=\bar{R}^{-1} R \bar{R}$. One can check that this mapping amounts to a rotation of the orthogonal axes $\mathbf{x}, \mathbf{y}$, and $\mathbf{z}$.
} 
in Figure 2.
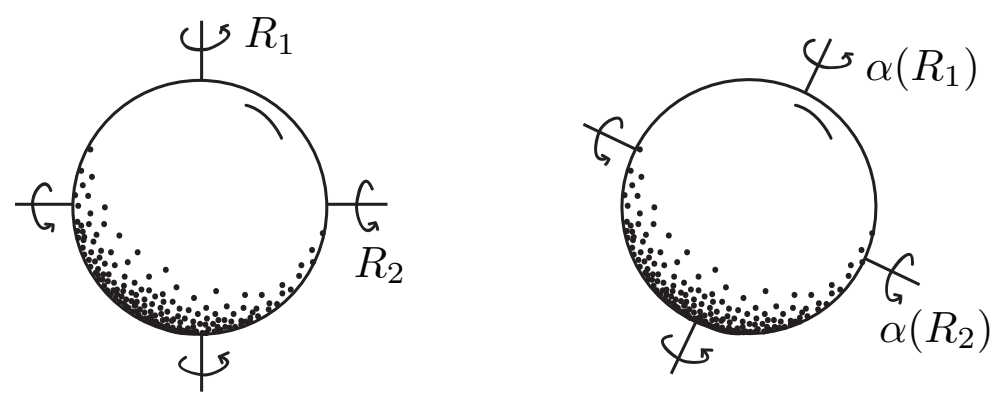

Figure 2: For a given presentation of $S O(3)$, an inner automorphism $\alpha$ sends each rotation (left) to its corresponding rotation about a different axis (right).

This example illustrates vividly how Aut $S O(3)$ really is the symmetry group of $S O(3)$. It is the group whose canonical action leaves any instantiation of $S O(3)$ invariant. Now, on the approach to structural realism provided by Wigner's legacy, we elevated the rotation group $S O(3)$ to a privileged metaphysical status. In that discussion, the thing being rotated was something like an electron shell. Now, in this new case, it seems we should elevate Aut $S O(3)$ instead. The only apparent difference is that, when we elevate the status of Aut $S O(3)$, the thing getting rotated is a presentation of $S O(3)$ itself.

Moreover, all of the virtues of elevating $S O(3)$ seem to carry over when we elevate the metaphysical status of Aut $S O(3)$ instead. Note that in both cases, some important properties are left invariant under the action of the group (that is, both can be called 'symmetry' groups). In the case of the electron shell, they are the properties deriving from the total angular momentum $j$. In the case of $S O(3)$ itself, they are the properties deriving from the group structure ${ }^{16}$. Note also that both can be taken as the basis for a construction in which the rest of quantum theory is recovered. The only difference is, the group Aut $S O(3)$ is 'one level more abstract,' so that this construction begins by constructing an invariant group $S O(3)$, and then proceeding as usual.

As French and Ladyman suggest, an infinite regress now threatens. In general, the group Aut $G$ will also admit an automorphism group. This gives rise to what is known as an automorphism tower ${ }^{17}$, given by

$$
G, \operatorname{Aut} G, \text { Aut Aut } G, \ldots
$$

\footnotetext{
${ }^{16}$ This is because each automorphism $\alpha \in$ Aut $S O(3)$ is a group isomorphism

${ }^{17}$ See Robinson (1996 408-415) for an introduction to the study of automorphism towers.
} 
As long as each successive automorphism group results in a distinct new group (more on this later), we can continue producing new, 'metaphysically fundamental' structures all the way up. Since this tower can be very high, the result is a bloated, very abstract ontology. Indeed, there are even groups for which the tower can be continued transfinitely ${ }^{18}$. And worse: it can also happen the groups of an automorphism tower cycle. For example, take the infinite dihedral group $D_{\infty}$ (which is a subgroup of any orthogonal group $O(m, n)$ ). While $D_{\infty} \nsucceq$ Aut $D_{\infty}$, it can be shown that $D_{\infty} \cong$ Aut Aut $D_{\infty}$ (Hulse 1970). So the nodes of this tower bounce back and forth between $D_{\infty}$ and Aut $D_{\infty}$. Now, which of a cycling pair of group structures should the structural realist choose?

Here is how the worry looks in the form of the above a dilemma. Horn 1. The advocate of GSR would like to place the original group $G$ at top of the metaphysical hierarchy. But there does not seem to be a well-motivated reason to choose $G$ over Aut $G$ (this is the subject of the next subsection). This pushes GSR to: Horn 2. We instead promote the highest automorphism group Aut $G$ in the tower, or else promote the 'whole shebang.' This introduces a tower's worth of 'lower down' groups into our ontology, and (as we will see) does not appear to satisfy the aims of structural realism.

In summary: group structural realism is forced to either risk arbitrariness (Horn 1), or else adopt an overly extravagant and uninformative account of reality (Horn 2).

Trying to overcome the first horn seems to be the first natural first choice here. For example, one might give theoretical reasons for preferring to elevate only the original group $G$ (this possibility will be addressed in Section 3.3). Or, one might suggest that elevating Aut $G$ doesn't do as good a job of satisfying the aims of structural realism, such as accounting for theory change. Given the close similarity between $G$ and Aut $G$ on the Wigner-approach to structural realism, this last suggestion seems unlikely. In the case of theory change, we know that Aut $G$ will be preserved whenever $G$ is, since the former is uniquely defined by the latter. But the example of theory change provides an even more interesting argument against the first horn: there is a precise sense in which an Aut $G$ actually does a better job than the original group $G$ at accounting for theory change. Let's have a brief discussion of why this is.

\footnotetext{
${ }^{18}$ We discuss one example below; examples may also be found in Collins (1978) and Hamkins (1998).
} 


\subsection{Higher Structures and Theory Change}

We saw in Section 2.3 that theory change can tend to favor a group $G$ over an individual object. The reason for this was that groups are often insensitive to a change in underlying set. As it turns out, this is just as much a reason for thinking that theory change favors Aut $G$ over $G$.

Here's another toy example: the rotational symmetry group of a triangle is the cyclic group $C_{3}$ of order three; the rotational symmetry group of a square is the cyclic group $C_{4}$ of order four. These groups are not isomorphic, but the automorphism groups Aut $C_{3}$ and Aut $C_{4}$ are ${ }^{19}$. To tell another just-so story: suppose some theory led us to posit the existence of a triangle, and we later discovered there is rather a square. We would have the symmetry group, as well as the object. But the automorphism group would be preserved under this theory change.
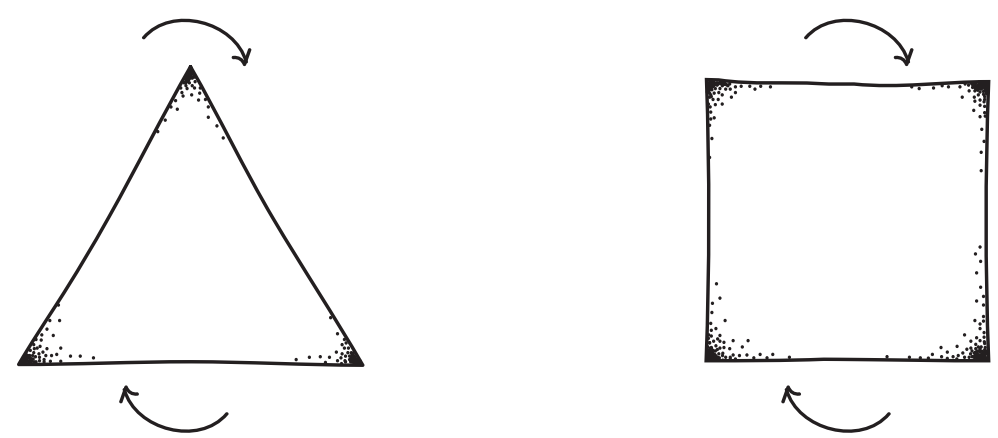

Figure 3: The rotational symmetry group of the triangle is not isomorphic to the rotational symmetry group of the square. However, their automorphism groups do turn out to be isomorphic.

Here is a theoretical argument suggesting this tendency to preserve an automorphism group, and not the original group, is very common indeed. Consider any theory in which the onedimensional group of rotations is a symmetry group. This includes theories involving Lorentz group $O(3,1)$, the homogeneous Galilei group $O(3)$, and both of the rotation groups that we have been discussing $(S O(3)$ and $S U(2))$. The following proposition suggests that, within such theories, favoritism towards an automorphism group is exceedingly common ${ }^{20}$ :

Proposition 1. Let F contain a subgroup isomorphic to $U(1)$, the one-parameter 'circle group' of rotations. Then $F$ contains infinitely many pairs of subgroups $G$ and $H$ such that $G \neq H$, and

\footnotetext{
${ }^{19} C_{3}$ and $C_{4}$ each admit just two automorphisms: the identity mapping, and the mapping that exchanges the generators. $C_{2}$ is the only group of order two (up to isomorphism), so it follows that Aut $C_{3} \cong C_{2} \cong$ Aut $C_{4}$.

${ }^{20}$ We provide a proof in the Appendix to this paper.
} 
Aut $G \cong$ Aut $H$.

Here's a just-so story to illustrate the proposition. Suppose some theory change demands that we change our commitment from one subgroup of the Lorentz group (call it $G$ ) to another subgroup (call it $H$ ). Our proposition says that there are infinitely many examples in which $G$ is not isomorphic to $H$, but their automorphism groups do turn out to be isomorphic. So, in many, many cases, Aut $G$ seems to stand a better change of surviving theory change than $G$ does.

If the advocate of GSR is motivated by theory change, it now seems that we have some reason to consider identifying the metaphysically fundamental group with Aut $G$, rather than with $G$ : the automorphism group is more apt to be preserved. This pushes us towards the second horn of the dilemma. But before we give up on this horn, let's discuss some other ways that a structural realist might try to promote $G$ over Aut $G$.

\subsection{Any Way Out for the Groupies?}

So far, we've observed that a 'higher structure' - such as an automorphism group - does not fit clearly into the cross-hairs of structural realism. Even a precise, seemingly sensible special case like GSR does not provide a sensible way to choose which structure to promote.

However, to be fair, structural realism was not developed with higher structures in mind. For example, higher automorphism structures are completely missed by the 'partial isomorphisms' approach to structural realism, which focuses on the way that structures get embedded as substructures into other structures ${ }^{21}$. In this section, we discuss the possibility of refining structural realism so as to consistently incorporate these higher structures. The bad news is that none of these possible refinements provide a satisfactory solution.

The problem of higher structures gets its force from this fact: if $S$ can plausibly occupy the top position in the structural realist hierarchy, then it seems at least as plausible that the structure of $S$ can occupy this position. So we are faced with a dilemma: should the structural realist choose the first horn, and seek some way to specify which is the structure of interest? Or, should she choose the second horn, and specify many or all of the higher structures?

\footnotetext{
${ }^{21}$ For a discussion of the partial isomorphism account of theory change, see (Bueno, French, and Ladyman 2002). See (French and Ladyman 2003) for an application of this view to structural realism.
} 
Choosing the first horn of the dilemma seems to require we choose between one of two strategies:

- find a way to promote only structures that have no non-trivial higher structures; or

- add some apparatus that chooses a structure $S$ over its higher structures.

Choosing the second horn of the dilemma apparently requires that we:

- allow that both $S$ and its structure (or perhaps just the latter) somehow be promoted to the top of the metaphysical hierarchy.

In Sections 3.3.1, 3.3.2 and 3.3.3 we treat each of these three options in turn.

\subsubsection{Chopping Down the Tower}

Not every structure has a non-trivial tower of higher structures. For example, $S_{3}$, the permutation group of three objects, has the property $S_{3} \cong$ Aut $S_{3}$. There is no need to choose between this group and its automorphism group, because the two are isomorphic. Unfortunately, most important groups in physics do not have this property. In particular, we have the following ${ }^{22}$ :

Proposition 2. Every special unitary group $S U(n)$, special orthogonal group $S O(m, n)$, orthogonal group $O(m, n)$, and kinematical group $O(m, n) \rtimes \mathbb{R}$ has a non-trivial automorphism tower.

Since almost all of the groups discussed so far lie within the scope of Proposition 2, this strategy is not very promising. However, one might still try to replace a given group with one that has a non-trivial automorphism tower, through the use of the following two propositions.

Theorem 1. [Cayley's Theorem] An arbitrary group $G$ can be embedded into Sym $G$, the symmetric group ${ }^{23}$ on $G$ (Robinson 1996 pp. 36).

Theorem 2. For every symmetric group $\operatorname{Sym} G$ on a finite group $G$ (where $|G| \neq 2$ or 6 ), Sym $G \cong$ Aut Sym G (Robinson 1996 pp. 415).

\footnotetext{
${ }^{22}$ We sketch a proof in the appendix to this paper.

${ }^{23}$ The symmetric group on $G$ is the group of bijections from $G$ to itself. For finite groups of order $n$, Sym $G$ is often denoted $S_{n}$, and is often called the permutation group.
} 
The idea is this: a structural realist might suggest that the metaphysically fundamental group isn't $G$, but rather the larger group $\operatorname{Sym} G$, which Cayley's Theorem tells us $G$ is embedded in. Theorem 2 then (mostly) guarantees that $\operatorname{Sym} G$ has a non-trivial automorphism tower, as long as $|G|$ is finite. Shirking $G$ in favor of its permutation group might thus provide one way out of the problem.

This idea doesn't get us very far. The restriction that $|G|$ be finite is very prohibitive. But even if there is an equivalent result for infinite groups, $\operatorname{Sym} G$ is still in a sense the worst possible choice for describing physical reality. Precisely because of Cayley's theorem, the symmetric groups contain all other groups as subgroups, and thus a zoo of structures that have nothing at all to do with the physical world. So I see now ay that the general adoption of Sym $G$ can be tenable.

\subsubsection{Playing Favorites}

It is thus unavoidable that a symmetry group may admit a tower of higher structures. Moreover, it is unlikely that one could in general pick out the 'top' of this tower, since there are groups with towers that extend transfinitely, as well as groups with towers that cycle. So, it seems the structural realist shouldn't hold out much hope to pick out the top of the tower. Can the structural realist provide some motivation for choosing the 'bottom' of the tower? That is, can one argue that $G$ is more fundamental than Aut $G$ and the other higher structures? I can imagine three non-arbitrary ways that the one might do this: by appealing to the real world, by appealing to the mathematics, and by appealing to the physics. None of them are very appealing.

First, one might argue that the metaphysically fundamental group is the one that is least abstractly removed from the real world. For example, in our discussion of angular momentum, $S O(3)$ seems to be the most significant group, because it acts directly an electron shell. On the other hand, Aut $S O(3)$ acts on $S O(3)$, which in turn acts on an electron shell. This seems to give us good reason to think that $S O(3)$ is more metaphysically significant than Aut $S O(3)$ : the former is less abstractly removed.

However, if our goal is to pick out what's metaphysically significant, this begs the question: removed with respect to what? The response cannot be: 'Removed with respect to the electron shell.' As discussed before, the advocate of GSR must provide an account in which groups are what underpin the properties of objects, and not the other way around. In particular, if $S O(3)$ 
is what provides individual objects with their properties, then it would be circular to turn around and use an individual object to pick out $S O(3)$. So we cannot use the notion of 'least abstractly removed' to pick out the metaphysically fundamental symmetry group. (For the same reason, the property of being 'most abstractly removed' won't work either.)

Second, one might try to pick out a group $G$ over Aut $G$ by arguing that the former is the most mathematically informative group. After all, $G$ fixes Aut $G$, but the converse is generally not true. So given an automorphism tower,

$$
G, \text { Aut } G, \text { Aut Aut } G \ldots,
$$

the group $G$ at the bottom of the tower might be identified as the most mathematically informative, because it uniquely determines the rest of the tower. As Armstrong might argue, if $G$ is what's fundamental, then the rest of the tower is a 'metaphysical free-lunch.'

But this suggestion won't work either, because if the 'bottom of the tower' exists, it is usually underdetermined. Note that there might well be some group $H$ such that Aut $H \cong G$. In this case, the above automorphism tower really looks like this:

$$
H, G, \text { Aut } G, \text { Aut Aut } G, \ldots
$$

There is no guarantee against an infinite regress here, by which the tower would have no bottom. But even worse, there is often more than one such $H$ that can extend the tower down in this way. In this case, we have no way to choose which 'bottom' of the tower is intended.

To see an example of this, let's return to the rotational symmetry group of the square $C_{4}$. This group is isomorphic to the automorphism group of $C_{5}$, the cyclic group of order five ${ }^{24}$. But $C_{4}$ is also isomorphic ${ }^{25}$ to the automorphism group of $C_{10}$. Therefore, we have two towers:

$$
C_{5}, C_{4} \text {, Aut } C_{4} \text {, Aut Aut } C_{4} \ldots \quad C_{10}, C_{4} \text {, Aut } C_{4} \text {, Aut Aut } C_{4} \ldots
$$

These towers have different groups 'at the bottom,' and we have no principled way to choose

\footnotetext{
${ }^{24}$ Here is a quick proof. Since $C_{5}$ is cyclic, Aut $C_{5} \cong \mathbb{Z}_{5}^{\times}$, the multiplicative group of units of the ring $\mathbb{Z}_{5}$ (Robinson 1996. Prop. 1.5.5). But 5 is prime, so $\mathbb{Z}_{5}^{\times} \cong C_{5-1}=C_{4}$. Therefore, Aut $C_{5} \cong C_{4}$.

${ }^{25}$ This follows from the proof of Proposition 1 in the appendix to this paper.
} 
between them. So it appears that choosing the group at the bottom of the tower brings us right back to where we started: we have multiple candidates for the role of 'metaphysically fundamental' structure, and no way to choose between them.

Finally, the structural realist might encourage us to accept a group $G$ simply because it is the group that is most naturally suggested by the physics. This idea is perhaps closest to the right attitude. Physics certainly prescribes a clear role for some groups, and not for others. For example, the rotation group $S O(3)$ is simply a fundamental consideration in the treatment of angular momentum in quantum systems. On the other hand, it is less clear what role Aut $S O(3)$ plays. Why not take this distinction between $S O(3)$ and Aut $S O(3)$ seriously?

If a natural physical attitude were the target, then the structural realist should have stopped at Wigner's legacy. This was clearly a fruitful episode in the history of physics. However, that simply doesn't translate into a fruitful metaphysics. As many structural realists have argued, the correct interpretation of these physical results is a separate question. Ladyman has hammered 'the impossibility of simply 'reading one's metaphysics off one's physics' (Ladyman 1998, pp. 419). French similarly argues 'theory itself provides no guide to ontology' (French 1999, pp. 204). The worry is presumably that, if we read too much into the physics, we might not end up with the correct structural realist hierarchy. Thus, structural realists are effectively barred from this seemingly natural scientific attitude.

If there are any other ways to coherently get around the first horn of the dilemma, then they are not forthcoming. So let's finally turn to the second horn, in which the entire tower - the whole shebang of structures - is elevated to 'metaphysically fundamental' status.

\subsubsection{Adopting the Whole Shebang}

Promoting the whole shebang of higher structures may be the most well-motivated option for the structural realist. The entire tower is certainly part of the available mathematical apparatus for describing the world. Quite simply, it all looks like structure. So why not take it all to be equally 'fundamental'?

For the structural realist motivated by theory change, choosing this way out is giving up the game. The idea was supposed to be that higher, more abstract structures are more disposed to be preserved when scientific theory changes. But adopting the whole shebang means adopting 
both the higher and the lower down structures. So, at least when it comes to theory change, the 'whole shebang' is only as safe as its least-abstract element. Moreover, once the entire tower has been thrown into our ontology, it's much less obvious why we don't just throw in all the available structures appearing in our theory - algebras, vector spaces, Hermitian forms, and so on. More structures means more sensitivity to change over time. So any advantage the structural realist had over its rival realist counterparts with respect to theory change would evaporate.

Another more homely worry about adopting the shebang is that it's just too wild. Whether or not an automorphism tower is finite, it still introduces a potentially enormous array of new group structures into the metaphysical hierarchy. And it still remains to be seen how a cycling tower of automorphisms fits into the hierarchy, as it seems impossible to call any point on such a tower more or less fundamental. However these worries are dealt with, they invariably seem to involve an overly extravagant ontology, which is both unmotivated and exceedingly complex. If this is what is needed to make sense of the reality behind the physics, then it seems we might be on the wrong track.

Perhaps this does not settle the issue. For example, one might hope that all compact Lie groups have well-behaved automorphism towers ${ }^{26}$. However, this seems an unlikely hazard, given the bad behavior of the infinite dihedral group $D_{\infty}$. As things currently stand, adopting the whole shebang does not appear to be a promising response.

\section{Conclusion}

The conclusions of this paper, if they are correct, can be taken in either a positive or a negative light. Critics of structural realism can see it as an extremely charitable criticism. Supporters of structural realism can see it as a back-handed contribution. What I hope to have shown in both cases is that there are two competing poles in working out a precise account of structural realism. On the one hand, we have a compelling account of the foundations of quantum theory in which group structure plays a central role. This account provides a great deal of insight into both the nature of measurable quantities, as well as into what is preserved as physical theory changes. On the other hand, we have

\footnotetext{
${ }^{26}$ Indeed, we already know that the Lorentz group $O(3,1)$ has a very short tower: Aut $O(3,1) \cong$ Aut Aut $O(3,1)$ (Michel 1967).
} 
the non-trivial difficulty of working out just what is meant by a 'metaphysical hierarchy,' and how a vast array of relevant theoretical structures fit into it.

This problem may yet turn out to be tractable, and structural realists are invited to try to overcome the difficulties we have posed here. However, by the lights of the current author, there is a more natural attitude to adopt. Namely: Wigner's legacy as shown itself be of deep importance and relevance; the structural realist hierarchy has not. It is possible to consistently support Wigner while avoiding this extra appendage, and so the latter may be more trouble than it's worth.

\section{Appendix}

Proposition 1. Let $F$ be a group with a subgroup isomorphic to $U(1)$, the one-dimensional group of smooth rotations. Then $F$ contains infinitely many pairs of subgroups $G$ and $H$ such that $G \varsubsetneqq H$, and Aut $G \cong$ Aut $H$.

Proof. Every cyclic group $C_{r}$ is a subgroup of $U(1)$, generated by a rotation of $2 \pi / r$. We prove that for every $C_{p}$ such that $p$ is an odd prime, Aut $C_{2 p} \cong \operatorname{Aut} C_{p}$. Then since $C_{2 p} \nsucceq C_{p}$, the proposition follows from the infinitude of the primes.

For any integer $n$, one can show that Aut $C_{n} \cong \mathbb{Z}_{n}^{\times}$, where $\mathbb{Z}_{n}^{\times}$is the group of multiplicative units of the ring $\mathbb{Z}_{n}$ (Robinson 1996 Prop. 1.5.5). In particular, Aut $C_{p} \cong \mathbb{Z}_{p}^{\times}$and Aut $C_{2 p} \cong \mathbb{Z}_{2 p}^{\times}$. But by the Chinese remainder theorem, if $n=p_{1} \cdot p_{2} \cdot p_{3} \ldots$ and each $p_{i}$ is a distinct prime, then

$$
\mathbb{Z}_{n}^{\times} \cong \mathbb{Z}_{p_{1}}^{\times} \times \mathbb{Z}_{p_{2}}^{\times} \times \mathbb{Z}_{p_{3}}^{\times} \times \cdots
$$

So $\mathbb{Z}_{2 p}^{\times} \cong \mathbb{Z}_{2}^{\times} \times \mathbb{Z}_{p}^{\times}$whenever $p$ is odd. But $\mathbb{Z}_{2}^{\times} \cong\{1\}$, the trivial group. So $\mathbb{Z}_{2 p}^{\times} \cong \mathbb{Z}_{p}^{\times}$, and hence Aut $C_{2 p} \cong \operatorname{Aut} C_{p}$.

Proposition 2. Every non-trivial special unitary group $S U(n)$, special orthogonal group $S O(m, n)$, orthogonal group $O(m, n)$, and kinematical group $O(m, n) \rtimes \mathbb{R}$ has a non-trivial automorphism tower.

Proof (sketch). Note that group $G$ is said to have a non-trivial automorphism tower iff $G \nsucceq$ Aut $G$. 
Each of the groups of Proposition 2 can be easily shown to have this property, with the help of the following lemma:

Helpful Lemma. Let $\zeta G$ be the center of a group $G$, and let Out $G$ be its outer automorphism group. If $|\zeta G| \neq \mid$ Out $G \mid$, then $G \neq$ Aut $G$.

In this sketch, we state the Helpful Lemma without proof. (For finite groups, it can be easily demonstrated using Lagrange's theorem; this result can easily be extended to the infinite case.) It can now be easily shown that for each of the symmetry groups of Proposition $2|\zeta G| \neq \mid$ Out $G \mid$, and so the Helpful Lemma may be applied.

Corollary 1. Every non-trivial $(n>1)$ special unitary group $S U(n)$ has a non-trivial automorphism tower.

Proof. Let $n>1$. It is well known that the center $\zeta S U(n)$ is a cyclic group generated by $\gamma I$, where $\gamma$ is the $n$th root of unity (Fendley 2001, pp. 124). Thus, $|\zeta S U(n)|=n$.

Now, turn to Out $S U(n)$. There are two cases to consider. First, let $n=2$. Then the only automorphism of Out $S U(n)$ is trivial, so $\mid$ Out $S U(n) \mid=1$. Second, let $n>2$. Then one can show that $\mid$ Out $S U(n) \mid=2$ (Froggatt and Nielsen 1991 pp. 154). In both cases, $|\zeta S U(n)| \neq$ $\mid$ Out $S U(n) \mid$. Therefore, by the Helpful Lemma, $S U(n) \neq$ Aut $S U(n)$.

Corollary 2. Every special unitary group $S U(n)$, special orthogonal group $S O(m, n)$, orthogonal group $O(m, n)$, and kinematical group $O(m, n) \rtimes \mathbb{R}$ has a non-trivial automorphism group.

Proof. The proof of these facts is straightforward, following a similar strategy of showing that $|\zeta G| \neq|\operatorname{Out} G|$, and then applying the Helpful Lemma. Proposition 2 then follows as a consequence.

\section{References}

Bain, Jonathan. 2000. "Against Particle/Field Duality: Asymptotic Particle States and Interpolating Fields in Interacting QFT (or: Who's Afraid of Haag's Theorem?)." Erkenntnis $53: 375-406$. 
Bargmann, V. 1954. “On Unitary Ray Representations of Continuous Groups.” Annals of Mathematics 59 (1): 1-46.

Bueno, Otávio, Steven French, and James Ladyman. 2002. “On Representing the Relationship between the Mathematical and the Empirical.” Philosophy of Science 69:497-518.

Collins, Donald J. 1978. “The Automorphism Towers of Some One-Relator Groups.” Proceedings of the London Mathematical Society 36 (3): 480-493.

Dorato, Mauro. 2000. “Substantivalism, Relationism, and Structural Spacetime Realism.” Foundations of Physics 30 (10): 1605-1628.

Eddington, Arthur S. 1958. The Philosophy of Physical Science. Ann Arbor: University of Michigan Press.

Fendley, P. 2001. "Integrable Sigma Models.” In Integrable Quantum Field Theories and Their Application, Proceedings of the APCTP Winter School, edited by C. Changrim Ahn, 108-178. Singapore: World Scientific Publishing Co. Pte. Ltd.

French, Steven. 1999. "Models and mathematics in physics: the role of group theory." In From Physics to Philosophy, edited by Jeremy Butterfield and Constantine Pagonis, 187-207. Cambridge: Cambridge University Press.

French, Steven, and James Ladyman. 2003. "Remodelling Structural Realism: Quantum Physics and the Metaphysics of Structure." Synthese 136:31-56.

—. 2008. "In Defense of Structural Realism.” November 2008 manuscript. Forthcoming in Scientific Structuralism, Bokulich and Bokulich (eds), Boston Studies in the Philosophy of Science, Boston University Press.

Froggatt, C. D., and H. B. Nielsen. 1991. Origin of Symmetries. Singapore: World Scientific Publishing Co. Pte. Ltd.

Gell-Mann, Murray. 1961. "The Eightfold Way: A Theory of Strong Interaction Symmetry." Report CTSL-20, California Institute of Technology.

Hamkins, Joel David. 1998. "Every Group Has a Terminating Transfinite Automorphism Tower.” Proceedings of the American Mathematical Society 126 (11): 3223-3226. 
Hulse, J. A. 1970. “Automorphism Towers of Polycyclic Groups.” Journal of Algebra 16:347398.

Kantorovich, Aharon. 2003. "The priority of internal symmetries in particle physics." Studies in History and Philosophy of Modern Physics 34:651-675.

—. 2006. "Partices vs. Structures: Weak Ontic Structural Realism.” Philsci-Archive, accessed 31-Jan-09, philsci-archive.pitt.edu/archive/00003068.

Ladyman, James. 1998. "What is Structural Realism?" Studies in History and Philosophy of Science 29 (3): 409-424.

—. Spring 2009. "Structural Realism.” In The Stanford Encyclopedia of Philosophy, edited by Edward N. Zalta.

Ladyman, James, and Don Ross. 2007. "Ontic Structural Realism and the Philosophy of Physics.” Chapter 3 of Every Thing Must Go: Metaphysics Naturalized, edited by James Ladyman and Don Ross, 130-189. New York: Oxford University Press.

Lévy-Leblond, Jean-Marc. 1967. "Nonrelativistic Particles and Wave Equations." Communications in Mathematical Physics 6:286-311.

Lyre, Holger. 2004. "Holism and structuralism in $U(1)$ gauge theory." Studies in History and Philosophy of Modern Physics 35:643-670.

Michel, Louis. 1967. "Relations entre Symétries Internes et Invariance Relativiste." Cargèse Lectures in Theoretical Physics. Gordon and Breach Science Publishers, 409-452.

Ne'eman, Yuval. 1961. "Derivation of strong interactions from a gauge invariance." Nuclear Physics 26:222-229.

Ne'eman, Yuval, and Shlomo Sternberg. 1991. "Internal Supersymmetry and Superconnections." In Symplectic Geometry and Mathematical Physics, edited by Donato et al., 326-344. Boston: Birkhäuser.

Psillos, Stathis. 2006. “The Structure, the Whole Structure and Nothing but the Structure?" Philosophy of Science 73:560-570.

Robinson, Derek J. S. 1996. A Course in the Theory of Groups. 2nd Ed. New York: SpringerVerlag New York, Inc. 
Sewell, Geoffrey. 2002. Quantum Mechanics and Its Emergent Macrophysics. Princeton: Princeton University Press.

Weyl, Hermann. 1950. The theory of groups and quantum mechanics. 2nd Ed. New York: Dover Publications, Inc.

Wigner, Eugene. 1931. Group Theory and its Application to the Quantum Mechanics of Atomic Spectra. New York: Academic Press (1959).

. 1939. “On Unitary Representations of the Inhomogeneous Lorentz Group.” Annales of Mathematics 40:149.

Worrall, John. 1989. "Structural realism: The best of two worlds?” Dialectica 43:139-165. 